\title{
Dysregulated Network of Immune, Endocrine and Metabolic Markers is Associated to More Severe Human Chronic Chagas Cardiomyopathy
}

\author{
Florencia González ${ }^{a}$ Silvina Villar ${ }^{a}$ Luciano D'Attilio ${ }^{a}$ Rodolfo Leivab \\ Julia Marquez ${ }^{a}$ Susana Lioic Juan Beloscar ${ }^{b}$ Oscar Bottasso ${ }^{a}$ \\ Ana Rosa Perez ${ }^{a}$
}

anstituto de Inmunología Clínica y Experimental de Rosario (CONICET-UNR), Rosario, Argentina; ${ }^{b}$ Cátedra de Cardiología, Facultad de Ciencias Médicas (UNR) y Servicio de Cardiología, Sección Chagas del Hospital Provincial del Centenario, Rosario, Argentina; ' Laboratorio Central Rosario, Facultad de Ciencias Bioquímicas y Farmacéuticas (UNR) y Hospital Provincial del Centenario, Rosario, Argentina

\section{Keywords \\ Chagas disease $\cdot$ Immuno-metabolism - Cortisol · Tumor necrosis factor- $a \cdot$ Interleukin- $6 \cdot$ Adipocytokines · Tumor necrosis factor-a receptor type- $1 \cdot$ ObR $\cdot$ Adiponectin receptor type-1}

\begin{abstract}
Individuals who are infected with Trypanosoma cruzi develop chronic Chagas cardiomyopathy (CCC), which is a complication involving a series of immune pathogenetic mechanisms, although an association between immune and metabolic alterations was more recently proposed. Accordingly, we investigated the immuno-metabolic response in chagasic patients and their possible influence on CCC pathogenesis. To this end, $T$. cruzi-seropositive (asymptomatic or with CCC) and sero-negative individuals were studied. Serum tumour necrosis factor (TNF)- $a$, interleukin (IL)- 6 , adipocytokines and the expression of their receptors in peripheral blood mononuclear cell (PBMC) were evaluated, together with other factors influencing the immune response. CCC patients showed major metabolic and hormonal abnormali-
\end{abstract}

\section{KARGER}

(c) 2018 S. Karger AG, Basel

E-Mail karger@karger.com

www.karger.com/nim ties, in parallel with increased IL-6 and leptin serum levels. TNF-a receptor s, leptin and adiponectin receptors (ObR and Adipo-Rs respectively), as well as PPAR- $\gamma$ expression in PBMCs from CCC patients were compatible with a counteracting response leading to an unfavourable immune-metabolic profile. These results suggest that persistently increased levels of immune-metabolic pro-inflammatory mediators along with the adverse endocrine anti-inflammatory response of CCC individuals, may contribute to the underlying mechanisms dealing with myocardial tissue damage.

C 2018 S. Karger AG, Basel

\section{Introduction}

Chagas disease, caused by the parasite Trypanosoma cruzi, is considered a major health problem in Latina America. However, in the last decade, migratory flows have spread this disease into the United States, Europe and Asia [1]. These studies estimated that the disease burden, measured by disability-adjusted life-years, is 7.5 times as great as that of malaria [2]. The infection is 
caused by vectorial, congenital or oral transmission, although transfusional or transplant transmission was also reported [3]. During vectorial or congenital transmission, the acute phase is usually asymptomatic, lasting $~ 2-3$ months. After that, patients evolve to a chronic infection, where many of them remain asymptomatic for their lifetime. However, $\sim 30 \%$ of the chronically infected individuals progress to evident disease, with the development of different degrees of cardiac or digestive disturbances [4]. It is estimated that chronic Chagas cardiomyopathy (CCC) causes 50,000 deaths per year [5].

During many years, chronic Chagas disease was mainly regarded as an autoimmune pathology. In the 1990s, the finding of T. cruzi DNA in myocardial inflammatory lesions by PCR, gave more support to the theory of parasite persistence as one of the causes of tissue and functional damage during the chronic stage. CCC is mainly characterized by focal inflammatory lesions with destruction of cardiomyocytes and driving nerve fibres, collectively involved in the development of cardiomegaly, heart block, arrhythmias, congestive heart failure or stroke [4].

Immune mechanisms linked to the development of CCC are highly heterogeneous and not yet fully understood, raising the need to investigate whether additional components, like metabolic and endocrine factors, are also involved in disease morbidity. It is known that the metabolic status is finely tuning by nutrition, energy expenditure and hormonal signals. The communication between general metabolism and the immune response also comprises a series of pleiotropic products able to modulate appetite, endocrine functions, energy expenditure and inflammation, lastly affecting the regulation of energy sources and immune activity. Pro-inflammatory cytokines like tumour necrosis factor (TNF)- $\alpha$ and interleukin (IL)-6 and adipose tissue derived components like adipocytokines are also involved in the regulation of food intake [6, 7]. Moreover, hormones like glucocorticoids and dehydroepiandrosterone (DHEA), which may influence both metabolic and inflammatory responses [8-11], should also be considered in this complex scenario.

In this regard, a previous study carried out in a small group of urbanized patients with chronic Chagas' disease, showed that the more severe forms of myocarditis coexisted with immune-endocrine imbalances and a trend to overweight [12]. Coincidentally, another study detected a higher prevalence of diabetes in chagasic women with chronic myocarditis than in the general population [13]. Moreover, a recent work in chronically $T$. cruzi-infected mice showed that an obesogenic context promotes the development of cardiovascular disorders [14].

In this context, recent studies revealed that inflammatory chronic diseases present complex metabolic facets, with alterations in the relationship between catabolic and anabolic factors affecting diverse tissues and organs. Whether the fat tissue plays a role in human Chagas disease progression or in the severity of CCC has not been properly elucidated. There is evidence on the possible contribution of adipose tissue to T. cruzi-induced pathology in mice, but many of them are focused in the acute phase of infection [15-19]. Nevertheless, when considering that the adipose tissue seems to be one of the main reservoirs of T. cruzi during the chronic phase of disease [20], the possibility that a disbalance between certain fat inflamed-derived metabolic factors (like TNF- $\alpha$, IL-6, leptin and adiponectin) as contributing to cardiomyopathy progression and severity, sounds likely. For instance, unbalanced adipocytokines were associated with the presence of sympathetic dysfunctions in patients with CCC [21]. Moreover, cortisol/DHEA ratios, rather than the individual concentrations seem to be more representative of disease activity $[11,12]$.

It follows that disturbances in metabolic circuits might contribute to the pathogenesis and aggravation of human CCC. Here, we investigated the metabolic status in patients with chronic Chagas disease and examined whether there is a relation between metabolic, immune and endocrine patterns with the severity of MCC.

\section{Materials and Methods}

\section{Patients}

The study population consisted of 242 individuals, separated into 190 T. cruzi chronically infected patients and 52 age-matched healthy volunteers (control) of both sexes, recruited at the Chagas Section of the Cardiology Service of Hospital Provincial del Centenario de Rosario, Argentina. None of these patients was under specific treatment (i.e., benznidazole or nifurtimox) nor had concomitant pathological disorders. Exclusion criteria comprised other cardiac disease, neuroendocrine disturbances, major metabolic diseases (as diabetes), immunological diseases, or treatment with hormones or immunomodulators. Control subjects were seronegative to T. cruzi-specific tests. The diagnosis of Chagas disease was based on at least 2 positive serological findings according recommendations of the WHO, together with clinical symptoms, chest X-ray and 12-lead resting electrocardiogram (ECG). Chronic chagasic patients were grouped as follows: Asymptomatic (Asy; $n=84$ ), with normal ECG and chest $\mathrm{X}$-ray. CCC patients were subclassified into 2 groups: (a) Mild-toModerate cardiac patients $(\mathrm{M}, n=55)$, with no congestive heart failure and ECG showing any of the following alterations: incomplete right bundle branch block (RBBB) or complete RBBB, first degree of 
atrioventricular block or non-life-threatening arrhythmias or a chest $\mathrm{X}$-ray cardiothoracic ratio $<0.55$; (b) Severe patients $(\mathrm{SEV}, n=51$ ), presenting major ECG pathological tracings, that is, complex ventricular arrhythmia or complete atrioventricular block; congestive heart failure and a chest X-ray cardiothoracic ratio $>0.55$. As part of the physical examination, different anthropometric measurements were recorded. The population recruitment approach fit well with a consecutive non-probabilistic sample, rendering a predominant number of females in all study groups.

\section{Simple ECG Score}

The systematic ECG recording in endemic areas has allowed the possibility to describe several aspects of the natural electrocardiographic history of CCC, like the time of appearance and types of alterations noticed [22]. Based on this description, we developed a simple ECG score according to the progressive degree of alterations ranging from early to late electrocardiographic findings: RBBB (score 1); left anterior hemiblock (score 2); left bundle branch block (score 3); supraventricular extrasystoles (score 4); ventricular extrasystoles (score 5); first degree of atrioventricular block (score 6); supraventricular tachycardia (score 7); non-sustained ventricular tachycardia (score 8); complex ventricular tachycardia (score 9); and complete atrioventricular block (score 10). We calculated a simple scoring consisting of the summation of these point scores. Afterwards, we tested the eventual association between individual ECG scores and metabolic parameters.

\section{Plasma Collection for Cytokine, Adipocytokines and HPA}

Axis-Linked Hormone Assays

Fasting blood samples from patients and control were collected between 8:00 and 10:00 a.m. Plasma was obtained from EDTA treated blood. Samples were centrifuged at 2,000 rpm during 30 min and then preserved at $-70^{\circ} \mathrm{C}$. Plasma cytokine concentrations were assessed using commercially available enzyme-linked immunosorbent assay kits: IL-6 (R\&D), TNF-a (BD OptEIA), adiponectin (Invitrogen) and leptin (Peprotech), following manufacturer's indications. Plasma cortisol and DHEA sulphate (DHEA-S) were measured in duplicate by electrochemiluminescence immunoassay (Roche Diagnostics).

\section{Metabolic Variables}

The HOmeostasis Model Assessment of Insulin Resistance (HOMA-IR) index was calculated as (glucose $[\mathrm{mg} / \mathrm{dL}] \times$ insulin $[\mu \mathrm{UI} / \mathrm{mL}]) / 405$. Fasting glycaemia was evaluated by the glucoseoxidase assay (Wiener Lab.), whereas fasting insulin levels were assayed by the electrochemiluminescence method (Roche Diagnostics). Body mass index (BMI) was calculated as weight $/$ height $^{2}$ $\left(\mathrm{kg} / \mathrm{m}^{2}\right)$. Glycosylated haemoglobin and the lipid profile were determined by routine laboratory methods.

Metabolic Syndrome was defined using the International Diabetes Federation criteria $[23,24]$. They take into account central obesity as a prerequisite risk factor for metabolic syndrome, which can be easily assessed using waist circumference (male: $\geq 94 \mathrm{~cm}$; female: $\geq 80 \mathrm{~cm}$ ) or BMI $\geq 30 \mathrm{~kg} / \mathrm{m}^{2}$, plus 2 of the following factors: raised fasting plasma glucose $(>100 \mathrm{mg} / \mathrm{dL})$, high level of triglycerides $(>150 \mathrm{mg} / \mathrm{dL})$; reduced high density lipoprotein (HDL) cholesterol ( $<40 \mathrm{mg} / \mathrm{dL}$ in males and $<50 \mathrm{mg} / \mathrm{dL}$ in females) and increased blood pressure (systolic blood pressure $>130$ or diastolic blood pressure $>85 \mathrm{~mm} \mathrm{Hg}$ ), or specific treatments for any of these alterations.

Immuno-Metabolic Alterations in

Chronic Chagas Disease
Mononuclear Cell Isolation

Peripheral blood mononuclear cells (PBMCs) were obtained from fresh EDTA-treated blood. The cell suspension was layered over a Ficoll-Paque-PLUS (GE-Healthcare) gradient and centrifuged at $400 \mathrm{~g}$ for $30 \mathrm{~min}$ at $25^{\circ} \mathrm{C}$. The buffy coat was washed and re-suspended in RPMI 1640 (Gibco) and $8 \times 10^{6}$ cells and re-suspended in TRI-Reagent ${ }^{\circledR}$ (MRC) for RNA isolation and stored at $-80^{\circ} \mathrm{C}$ until used.

\section{RNA Isolation, cDNA Synthesis and $q P C R$}

Total RNA was isolated using TRI-Reagent ${ }^{\circledR}$ according to the manufacture's recommendations. The level of mRNA expression was determined by RT-qPCR. cDNA was synthesized from $2 \mathrm{ng}$ of total RNA using M-MLV reverse transcriptase (Invitrogen) and specific primers for TNF- $\alpha$ receptor type-1 (TNF-R1), TNF-R2, ObR, Adipo-R1, Adipo-R2 and PPAR- $\gamma$. PCR reactions were performed in a Stratagene MX3000P real-time PCR System (Stratagene), using the HOT FIREPol EvaGreen qPCRMix Plus with ROX master mix (SolisBiodyne), according to the manufacturer's recommendations. Data was normalized using cyclophilin A cDNA quantification.

Primer sequences used in this study were:

Cyclophilin A: F $5^{\prime}$-GGTCCTGGCATCTTGTCCAT-3'; R 5'-TTGCTGGTCTTGCCATTCCT-3'.

Adipo-R1: F 5'-CATCTACCTCTCCATCGTCTGTG-3'; R 5'-CATGAGGAAGAACCAGCCCATC-3'.

Adipo-R2: F 5'-CAGATAGGCTGGTTGATGCTGA-3'; R 5'-GCCGATCATGAAACGAAACTCC-3'.

ObR (Include all ObR isoforms): F 5'-GCCAGTCTTTCCAGAGAATAACCT-3'; R 5'-CAACTGTGTAGGCTGGATTGCT-3'.

PPAR- $\gamma$ (Include PPAR- $\gamma 1$ y PPAR- $\gamma 2$ isoforms): $\mathrm{F}$ $5^{\prime}$-TTTCAGAAATGCCTTGCAGTGG-3'; R $5^{\prime}$-CTTTCCTGTCAAGATCGCCCTC-3'.

IL-6R: F 5'-ATGTTGTTTGTGAGTGG-3'; R 5'-GACGCACATGGACACTATGTAGA-3'.

TNF-R1: F 5'-AGAGCCTAGACACTGATGACC-3' ${ }^{\prime}$ : R 5' -AGCATGCTGTATTGCGCCT-3'

TNF-R2: F 5' -ACTTCGCTCTTCCAGTTGGACT-3'; R 5'-AG GGCTTCTTTTTCACCTGGG-3'

\section{Statistical Analysis}

Parametric (analysis of variance followed by Tukey or Student $t$ test) or non-parametric tests (Kruskall-Wallis followed by Bonferroni test or the U the Mann-Whitney) were used depending on the specific variable characteristics. Pearson correlation analysis was also used. Categorical variables were analysed by the $\chi^{2}$. Given that many factors evaluated in this study showed a sexual dimorphism and age-related variations, both potential confounders were controlled by the unconditional logistic regression method. The level of significance was set at $p<0.05$.

\section{Ethics Statement}

The study was conducted according to the Helsinki Declaration and the protocol was approved by the Institutional Ethics Committee of the Medical Faculty of National University of Rosario. Participants were enrolled and gave their informed consent before inclusion. The protocol was approved by resolutions No. 2001/2012 and $4977 / 2013$. 
Table 1. Characteristics of chronic Chagas disease patients and control individuals

\begin{tabular}{|c|c|c|c|c|c|}
\hline \multirow[t]{2}{*}{ Patients } & \multirow{2}{*}{$\begin{array}{l}\text { Co } \\
(n=52)\end{array}$} & \multirow{2}{*}{$\begin{array}{l}\text { Asy } \\
(n=84)\end{array}$} & \multicolumn{2}{|c|}{$\operatorname{CCC}(n=106)$} & \multirow[t]{2}{*}{$p$ value } \\
\hline & & & $\mathrm{M}(n=55)$ & $\operatorname{SEV}(n=51)$ & \\
\hline Age, years & $47.2 \pm 1.9$ & $45.3 \pm 1.4$ & $52.2 \pm 1.7^{*}$ & $52 \pm 1.7^{*}$ & \multirow[t]{2}{*}{$<0.05^{*}$ vs. Asy } \\
\hline Range, $\min -\max$ & $18-73$ & $18-76$ & $21-76$ & $18-79$ & \\
\hline Gender, M:F & $20: 32$ & $28: 56$ & $9: 46$ & $17: 34$ & \multirow[t]{3}{*}{$-\mathrm{ns}\left(\chi^{2}\right)$} \\
\hline Systolic blood pressure, $\mathrm{mm} \mathrm{Hg}$ & $120 \pm 1.7$ & $120 \pm 2.0$ & $130 \pm 2.7$ & $130 \pm 1.8$ & \\
\hline Diastolic blood pressure, $\mathrm{mm} \mathrm{Hg}$ & $70 \pm 1.8$ & $74 \pm 1.3$ & $77 \pm 1.4$ & $80 \pm 1.2$ & \\
\hline
\end{tabular}

Co, control; Asy, asymptomatic; CCC, chronic Chagas cardiomyopathy; M, mild to moderate CCC; SEV, severe CCC, M:F, masculine/feminine number of individuals; min-max, minimum value-maximum value. Age and blood pressure data was expressed as mean $\pm S E$ of the mean. $\chi^{2}$, chi-squared test.

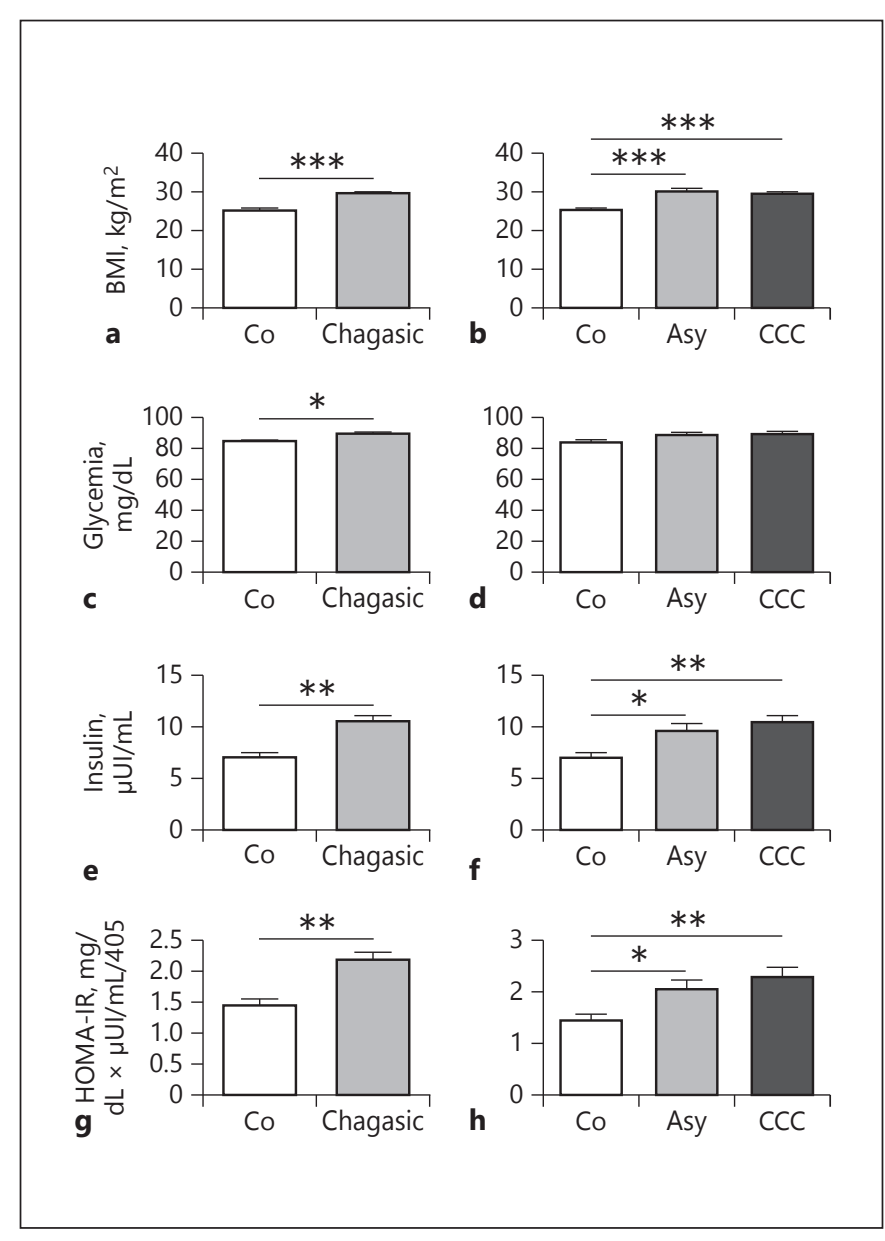

Fig. 1. Metabolic parameters in patients with Chagas disease in relation to healthy individuals. All parameters were evaluated depending on the presence (chronic Chagas cardiomyopathy [CCC]) or absence of cardiac pathology (Asy). a, b Body mass index (BMI), calculated as $\left([\mathrm{kg}] /\right.$ height $\left.^{2}[\mathrm{~m}]\right)$; c, d glycemia; e, f insulin; $\mathbf{g}, \mathbf{h}$ HOmeostasis Model Assessment of Insulin Resistance (HOMAIR). The results are expressed as mean \pm SEM. ${ }^{*} p<0.05$; ${ }^{* *} p<$ $0.01 ;{ }^{* * *} p<0.001$.

\section{Results}

\section{Study Population}

The characteristics of chronic Chagas disease patients and healthy controls are shown in Table 1 . All individuals were recruited at the Chagas Section of the Cardiology Service of Hospital Provincial del Centenario, Rosario, Argentina. Although the mean age was slightly higher in CCC patients than in the Asy ones (mostly due to the natural disease progression), age ranges did not differ among groups. There were more women than men in the total sample, but their frequency in each group was simi$\operatorname{lar}\left(\chi^{2}\right.$ test $)$. Among M patients, RBBB and LAH were the most prevalent ECG findings, while in SEV cases, $56 \%$ of them showed atrioventricular block (online suppl. Table 1; for all online suppl. material, see www.karger.com/ doi/10.1159/000491699).

\section{Metabolic Parameters in Individuals with Chronic Chagas Disease}

In the study population, individuals with chronic Chagas disease had a higher BMI than healthy individuals (Fig. 1a). In fact, $42 \%$ of total chagasic individuals presented BMI values compatible with obesity. This difference remained significant when chagasic individuals were sub-classified based on the presence or absence of myocardiopathy (Fig. 1b), with no differences between Asy and SEV cases (data not shown). A similar trend was observed in the waist/hip ratio (control $=0.84 \pm 0.0$, Asy $=0.88 \pm 0.1^{*}, \mathrm{CCC}=0.90 \pm 0.1^{*} ; p<0.05$ vs. control). We also observed differences in glycemia between control and chagasic individuals (Fig. 1c, d), more noticeable in SEV patients that showed the highest blood glucose values (control $=84.2 \pm 1.5$; Asy $=88.7 \pm 1.7$, $\mathrm{M}=88.8 \pm 2.4, \mathrm{SEV}=90.1 \pm 2.5,{ }^{*} p<0.05$ vs. control). 
Table 2. ECG score according to the body mass index categories of chronic chagasic patients with cardiomyopathy

\begin{tabular}{lcccc}
\hline ECG score & BMI $<25$ & BMI 25-30 & BMI 30-35 & BMI $>35$ \\
\hline M & $3(2-3.25)$ & $3(2.75-5)$ & $4(2-6)$ & $5(1.5-6.75)$ \\
SEV & $11(9-14)$ & $10(10-11)$ & $10(8.5-13.5)$ & $11(7.75-15.25)$ \\
\hline
\end{tabular}

ECG, electrocardiogram; BMI, body mass index; M, Mild to Moderate cardiomyopathy; SEV, Severe cardiomyopathy. ECG scores are expressed as median and 25-75 interquartile range.

Logistic adjustments indicated that glycaemia was not influenced by the BMI, or age, irrespective of the group. Although in our studies diabetic individuals were excluded, values of glycosylated haemoglobin in SEV patients were above the upper normal limit and statistically different from control values (control $=5.5 \pm 0.1$; Asy $=5.6 \pm 0.1 ; \mathrm{M}=5.6 \pm 0.1: \mathrm{SEV}=6.1 \pm 0.1^{*} p<0.05$ vs. control). This difference remained significant upon BMI adjustment. Unlike glycaemia, insulin levels were clearly increased in chagasic patients (Fig. 1e), either in Asy or CCC patients (Fig. 1f, $p<0.05$ respect to control), without differences between $M$ and SEV. Differences in insulin levels disappeared upon adjusting for the BMI. The same was true when analysing HOMA-IR, whose values in Asy and CCC patients appeared above those from control (Fig. 1g, h). Again, such differences became insignificant after adjusting for the BMI. Reported differences were also unmodified by age- or sex-adjustments. It follows that insulin and HOMA-IR alterations of chagasic individuals may be linked to the increased BMI. It also seems clear that regulation of glycemia requires a greater insulin secretion, resembling to some extent a pre-diabetic situation. Also, $\sim 30 \%$ of chagasic individuals presented metabolic syndrome as defined by the International Diabetes Federation criteria. Correlation analyses within the whole sample of CCC patients showed no relation between ECG score and the BMI. Nevertheless, when SEV patients were excluded, the BMI rise of M patients was associated with the progression of pathological ECG findings (Table 2), suggesting that a low but active systemic inflammation may favour the initial myocardial lesions that compromise the cardiac conduction system. Lipid profile analyses showed that although values oscillated within the normal range, $\sim 30 \%$ of chagasic patients showed increased amounts of triglycerides $(\geq 150 \mathrm{mg} / \mathrm{dL})$ or lower levels of HDL ( $\leq 40 \mathrm{mg} / \mathrm{dL}$ ) compared to control individuals, regardless of the degree of cardiac involvement (online suppl. Table 2).

Immuno-Metabolic Alterations in Chronic Chagas Disease
The bulk of these results suggested that in chagasic patients the immune response may be influenced by metabolic alterations.

\section{Immune-Metabolic Markers in Individuals with}

\section{Chronic Chagas Disease}

Next, we evaluated two key cytokines involved both in infection control and myocardial damage: IL-6 and TNF- $\alpha$. As expected, levels of circulating IL- 6 were higher in chronic chagasic patients than in control (Fig. 2a), this difference being due at the expense of the CCC group (Fig. 2b), regardless of age or BMI. Changes in TNF- $a$ moved in the same direction (Fig. 2c, d), although the increased levels displayed by CCC patients did not reach the levels of statistical significance. Adjustments for BMI, sex or age could not be applied because the variable deviated from the normal distribution. DHEA-S values in chagasic individuals situated below the control counterparts (Fig. 2e), being even lower in the CCC group (Fig. 2f). The same overall difference was seen when DHEA-S levels were age-adjusted. Further separation of patients by sex followed by age adjustment revealed that decreased DHEA-S levels remained statistically significant only in SEV women $(p<0.05)$. Chagasic individuals also showed a slight but significant hypocortisolemia in relation to control, irrespective of the clinical form (Fig. 2g, h), suggestive of a relative adrenal insufficiency. The cortisol/ DHEA-S ratio appeared increased in chagasic patients (Fig. 2i), but differences were only significant when comparing CCC patients with control and Asy cases $(p<0.05$ and $p<0.005$, respectively; Fig. $2 \mathrm{j}$ ). The approach of separating by sex and adjusting according to age rendered differences insignificant. Compared to control, leptin levels were significantly higher in individuals with chronic Chagas disease (Fig. 2k), mostly driven by the CCC group (Fig. 2l). Upon adjustment for BMI, such differences remained significant in women $(p<0.05)$, but were no longer observed in male patients. The trend of chagasic patients to show decreased adiponectin levels (Fig. $2 \mathrm{~m}$ ), at- 


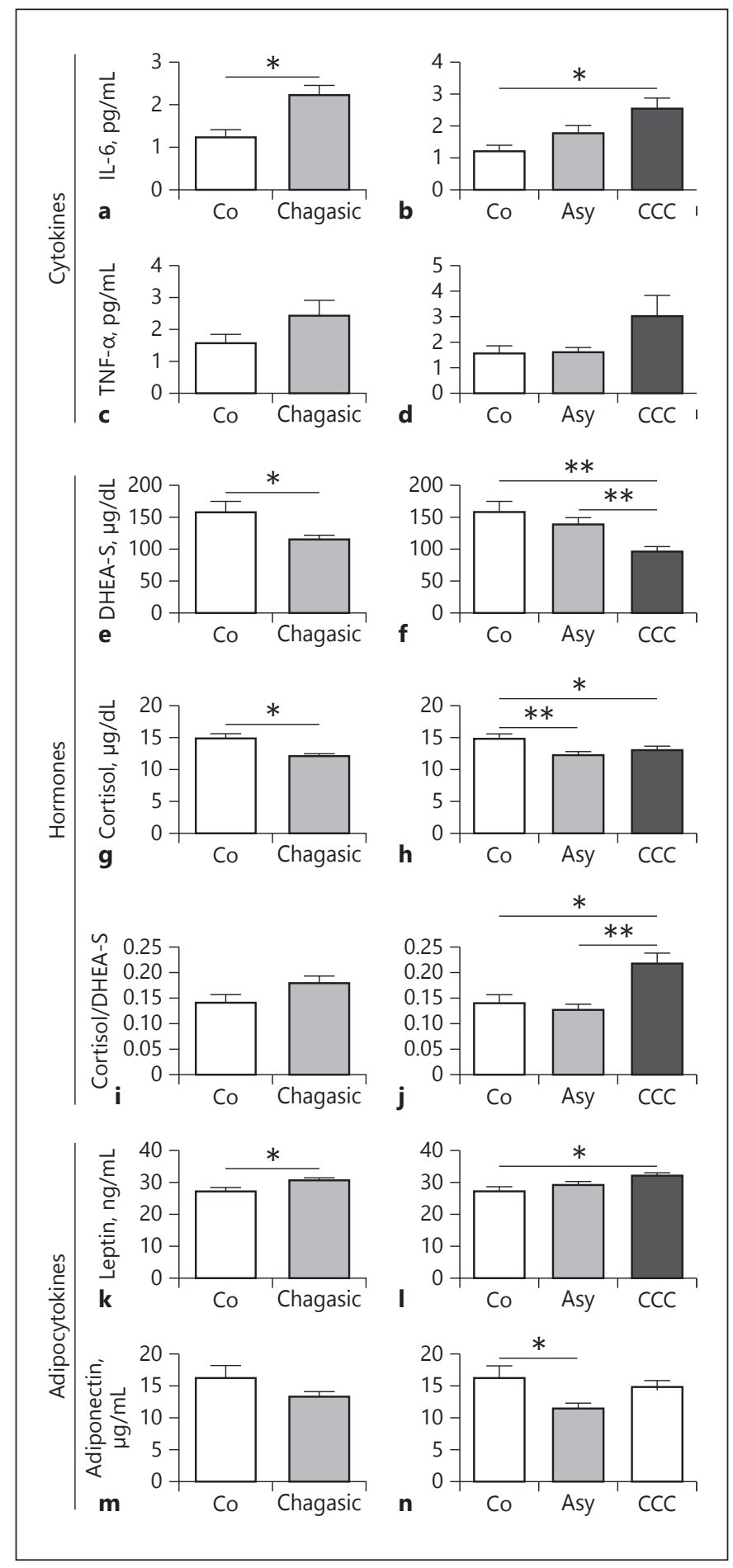

Fig. 2. Plasma levels of immuno-metabolic mediators in healthy and chagasic patients subdivided according to myocardial involvement. Cytokines: interleukin (IL)-6 (a, b); tumor necrosis factor (TNF)- $\alpha$ (c, d); hormones: DHEA-S (e, f); cortisol (g, h); cortisol/ DHEA-s $(\mathbf{i}, \mathbf{j})$; adipocytokines $(\mathbf{k}-\mathbf{n})$. The results are expressed as mean \pm SEM. ${ }^{*} p<0.05 ;{ }^{* *} p<0.01$. tained statistical significance in the case of Asy cases $(p<$ 0.05 vs. control; Fig. $2 \mathrm{n}$ ), with differences following BMI adjustment remaining significant only among women $(p<0.05)$.

\section{Expression of Immune and Immuno-Metabolic}

Related Receptors in Peripheral Mononuclear Cells

from Patients with Chronic Chagas Disease

To get some insight into the ability of immunocompetent cells to respond to circulating cytokines and adipocytokines, we further evaluated the expression of Adipo-R1, Adipo-R2, leptin receptor (ObR, all isoforms), IL-6R, TNF-R1 and TNF-R2 in PBMC. These assays were performed in fewer individuals because of operational issues. We also evaluated the expression of PPAR- $\gamma$, since its activation has modulatory effects on the immune and metabolic response of immune cells by inhibiting the release of inflammatory mediators, that is, TNF- $\alpha$ and leptin [25, 26], in addition to inhibiting the activation of T lymphocytes [27]. The present analysis indicated that PPAR- $\gamma$ transcripts tend to be increased in CCC individuals, without reaching the level of statistical significance (Fig. 3a). Interestingly, a significant increase in the expression of Adipo-R1 and Adipo-R2 was seen in CCC patients (Fig. 3b, c), whereas ObR transcripts were found sharply decreased in the same group (Fig. 3d). The assessment of IL-6R expression revealed no between-group differences (Fig. 3e). PBMC from chagasic patients had an increased expression of TNF-R1 and TNF-R2 respect to control, statistically significant in the case of TNF-R2 (Fig. 3f, g). The bulk of data suggests that immunocompetent cells tend to counterbalance the reported immuno-metabolic alterations.

\section{Discussion}

Currently, there is vast evidence pointing out that excessive weight gain, inflammation and the endocrine and metabolic disorders are closely related with the immune phenomena impacting at the cardiac level. Until a few years ago, the impact that this set of variables could have had on the severity of CCC was poorly appreciated. Nevertheless, some evidence from laboratory animals and human studies suggests that metabolic alterations triggered by the western lifestyle can influence the progression of Chagas disease $[14,19]$.

Within this setting, we have proceeded to evaluate a substantial number of urbanized and chronically-infected chagasic patients, to show that a considerable amount of them presented overweight and, in some cases, even 


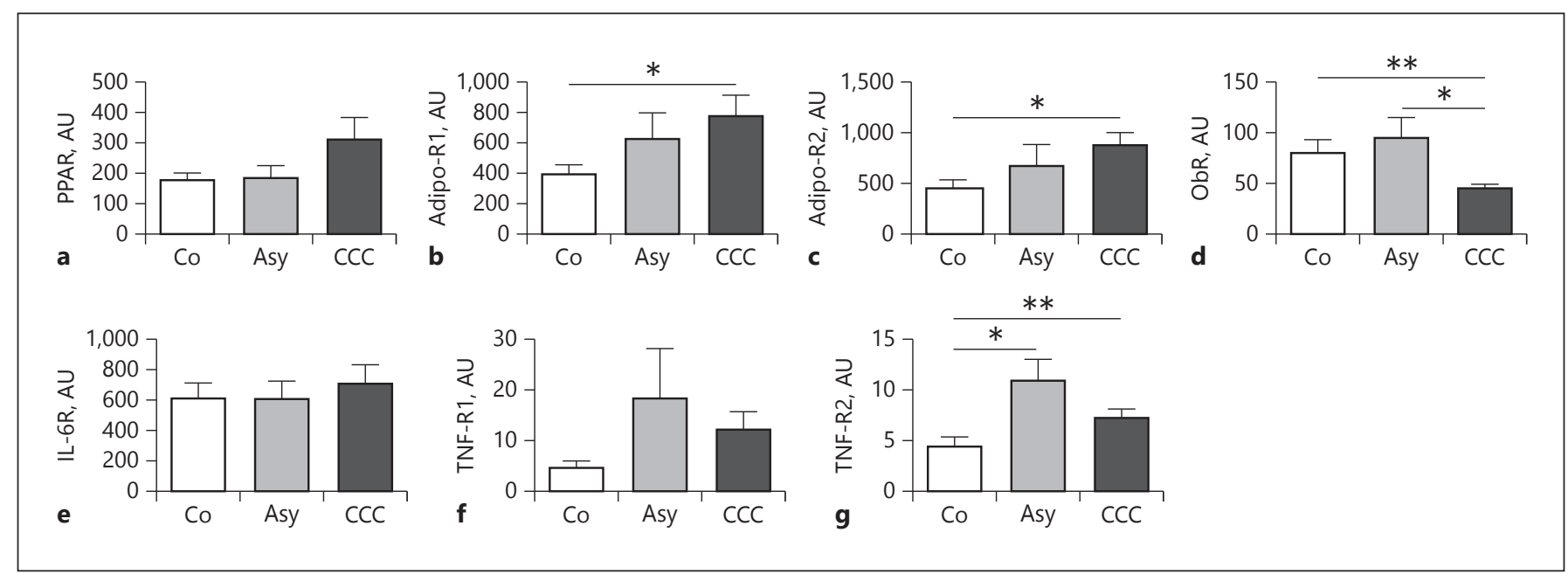

Fig. 3. Expression of immuno-metabolic-related receptors in peripheral mononuclear cells from patients with chronic Chagas disease: a PPAR- $\gamma$. b Adiponectin receptor type-1 (Adipo-R1). c Adiponectin receptor type-2 (Adipo-R2). d Leptin receptor (ObR). e IL-6 receptor (IL-6R). f TNF- $\alpha$ receptor type-1 (TNF-R1). g TNF- $\alpha$ receptor type-2 (TNF-R2). The results are expressed as arbitrary units and shown as mean \pm SEM $(n=15 /$ group, selected at random). ${ }^{*} p<0.05{ }^{* *} p<0.01$. obesity. Similarly, it has been reported that chagasic individuals residing in non-endemic areas of Brazil also manifested overweight [28], likely due to changes in their eating habits when migrating from endemic areas to large cities [29]. Within this setting, present patient series had increased insulin, triglycerides, HOMA-IR index in presence of lower levels of HDL likely compatible with a prediabetic situation, which in turn may increase the risk for cardiovascular disorders.

Overweight and obesity have a strong impact on the immune response, both being considered chronic lowgrade inflammation states $[30,31]$. In these conditions, adipocyte hypertrophy not only leads to the recruitment of inflammatory cells but also decreases Treg cells [3235 ], in addition to promoting IL- 6 and TNF- $\alpha$ secretion by macrophages and adipocytes $[35,36]$. The decreased presence of HDL may also favour the chronic inflammatory state given the anti-inflammatory properties of this lipoprotein. Since some of these abnormalities are present in CCC patients, such alterations are likely to underlie the pathogenesis of cardiomyopathy. Interestingly, we observed an association between the BMI and the ECG score - mirroring the degree of cardiac damage - in $M$ patients but not in SEV. Perhaps differences in the natural evolution of myocardial damage may be due to the initial focal inflammatory process, with the severe and well-established ECG alterations being more linked to irreversible cardiac damage because of the protracted fibrosis [22]. Accordingly, our results may implicate that an en-

Immuno-Metabolic Alterations in Chronic Chagas Disease hanced inflammatory background resulting from overweight is likely to favour the early development and establishment of cardiac lesions in chagasic patients.

Moving to adrenal steroid results, the increased cortisol/DHEA-S ratio seen in patients with CCC is reminiscent of the abnormal cortisol/DHEA-S relationship reported in non-chagasic heart failure [37] probably because of the stress resultant from this condition. Although BMI adjustment rendered the cortisol/DHEA-S insignificant, the fall in DHEA-S together with the slightly reduced cortisol levels of CCC patients points out to a partial deficiency of them to cope with the protracted inflammatory response and the subsequent myocardial affectation. Studies in the context of acute T. cruzi experimental infection demonstrated that administration of DHEA exerted a clear protective effect [38], whereas glucocorticoid blockade increased mortality [39].

As regards to cardiovascular disorders, obesity decreases adiponectin secretion and increases leptin resistance $[40,41]$, which are known to favour the occurrence of ventricular hypertrophy, heart failure, atrial fibrillation, the activation of pro-fibrotic pathways and the ensuing heart fibrosis [42]. Reinforcing this view, adiponectin deficient mice display a cardiomyopathic phenotype, with insulin resistance and obesity increasing the mortality associated to acute T. cruzi infection $[41,43]$.

In general terms, our series of chagasic patients had a slight hyperleptinemia without major changes in adiponectin levels. At variance with our findings, some authors 
Fig. 4. Immune-metabolic imbalances that may favour the establishment of chronic chagasic cardiomyopathy. The graph summarizes the complex and intricate network of immuno-endocrine and metabolic pathways during chronic Chagas disease.

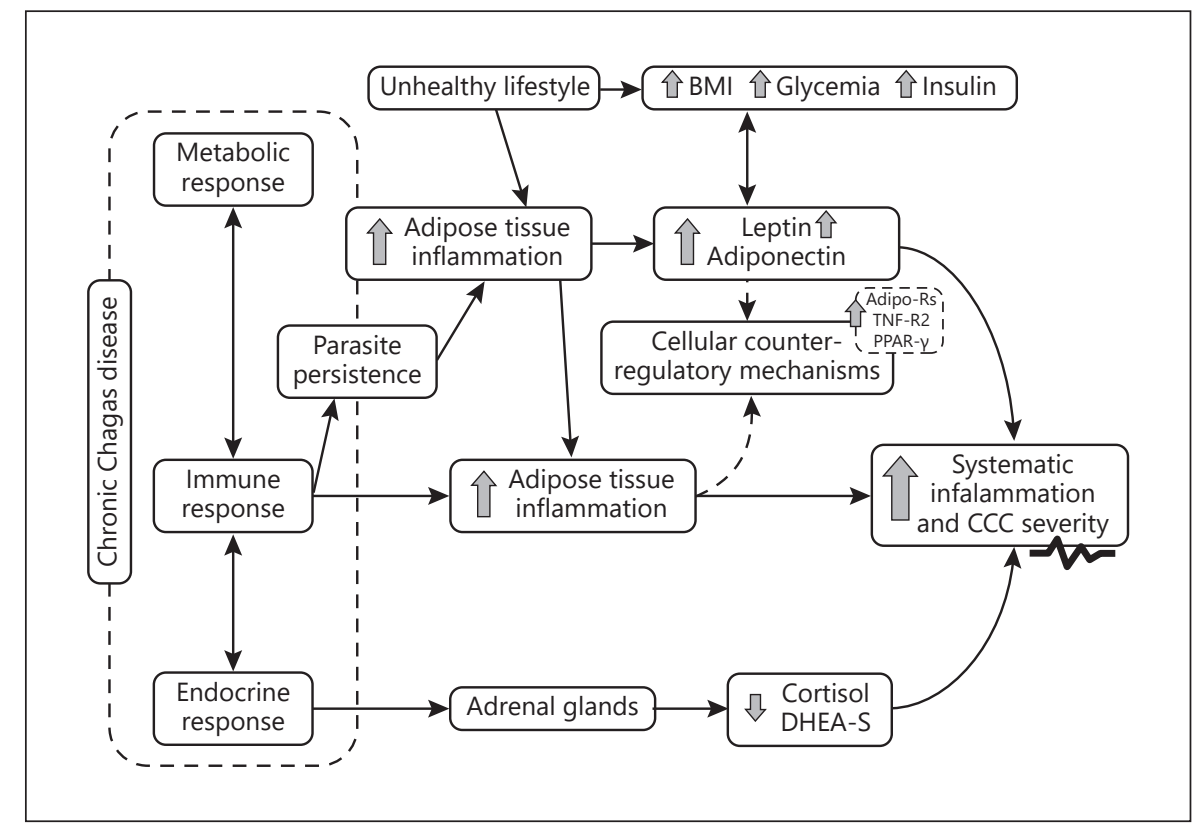

[21] reported decreased leptin levels in chagasic patients with congestive heart failure, whereas another study [44] showed no differences between CCC patients and controls. In both studies, however, the classification of myocardial involvement was different from the present one $[21,44]$. In line with our findings, leptin levels were found increased in non-chagasic individuals with chronic heart failure as a reflection of the proposed hypertrophic effects of leptin on cardiomyocytes $[45,46]$. Since heart enlargement is a common feature of CCC, a detrimental role of increased leptin levels in this regard cannot be ruled out [47]. As a non-mutually exclusive possibility, augmented leptin levels may be a reflection of increased inflammation, particularly in SEV patients, acting to account for the hormonal alterations of CCC patients because of its inhibiting effects on the production of adrenal androgens and cortisol [48].

The unchanged adiponectin levels seen in our patients, contrast with data reporting an autonomic dysfunctionrelated hyperadiponectinemia in a dissimilar subgroup of patients with advanced heart failure [21]. Although our CCC patients failed to show such an increase, they did exhibit an enhanced expression of adiponectin receptors, suggesting the presence of compensatory mechanisms to dampen inflammation. In the same sense, patients with chronic non-infectious inflammatory diseases display an increased expression of Adipo-Rs in their PBMCs [49, 50]. The same seems to apply for the expression of other receptors involved in anti-inflammatory functions. While the inflammatory effect of TNF- $\alpha$ is primarily mediated by TNF-R1, in systemic inflammatory diseases, TNF-R2 was shown to mediate anti-inflammatory functions by promoting suppressor T-cell populations [51, 52]. PPAR- $\gamma$ expression, which in our CCC patients displayed a tendency to be elevated, was found enhanced in immunocompetent cells from septic patients [53, 54]. Interestingly, PPAR- $\gamma$ agonist administration to T. cruzi-infected mice diminished heart inflammation and fibrosis [55], indicating that the anti-inflammatory pathways triggered by PPAR- $\gamma$ activation may ameliorate myocardial damage. Concerning ObR, its decreased expression in PBMCs of CCC patients may be mirroring the establishment of down-regulatory mechanisms, in terms of inflammationmediated tissue damage, as seen in chronic viral hepatitis [56]. Although cells infiltrating the myocardium may present another pattern of receptor expression due to in situ generated signals, the prolonged increase in immune and metabolic pro-inflammatory mediators along with the scarce endocrine control of inflammation likely to occur in CCC, emerge as contributing factors for the mechanisms underlying myocardial damage (summarized in Fig. 4).

In translating to the clinical field, present data suggests that overweight/obesity and its immuno-metabolic consequences must be addressed when evaluating individuals with Chagas disease, particularly when considering the increasing presence of this comorbidity among them. 


\section{Acknowledgements}

We thank the medical staff of the Cardiology Section from Hospital Provincial del Centenario de Rosario for their assistance and Wiener Lab., that provided glucose oxidase assay kits. This work was supported by grants provided by Argentinean funding institutions: CONICET (PIP 0641), ANPCYT (PICT 2013-1892) ASACTeI (2010-049-12) and SECYT-UNR (1MED410).

\section{Disclosure Statement}

The authors declare that they have no conflicts of interest to disclose.

\section{References}

1 Pinto Dias JC: Human chagas disease and migration in the context of globalization: Some particular aspects. J Trop Med 2013;2013: 789758.

2 Longo DL, Bern C: Chagas' disease. N Engl J Med 2015;373:456-466.

3 Coura JR: The main sceneries of Chagas disease transmission. The vectors, blood and oral transmissions - a comprehensive review. Mem Inst Oswaldo Cruz 2015;110: 277-282.

4 Rassi A, Rassi A, Marin-Neto JA: Chagas disease. Lancet 2010;375:1388-1402.

5 WHO Expert Committee: Control of Chagas Disease: Second report of the WHO expert committee. World Heal Organ - Tech Rep Ser 2002;1-99.

6 Johnson RW: Immune and endocrine regulation of food intake in sick animals. Domest Anim Endocrinol 1998;15:309-319.

7 Rondinone CM: Adipocyte-derived hormones, cytokines, and mediators. Endocrine 2006;29:81-90.

8 Karbowska J, Kochan Z: Fat-reducing effects of dehydroepiandrosterone involve upregulation of ATGL and HSL expression, and stimulation of lipolysis in adipose tissue. Steroids 2012;77:1359-1365.

9 Karbowska J, Kochan Z: Effects of DHEA on metabolic and endocrine functions of adipose tissue. Horm Mol Biol Clin Investig 2013;14: 65-74.

10 Dillon JS: Dehydroepiandrosterone, dehydroepiandrosterone sulfate and related steroids: their role in inflammatory, allergic and immunological disorders. Curr Drug Targets Inflamm Allergy 2005;4:377-385.

11 Straub RH, Paimela L, Peltomaa R, Schölmerich J, Leirisalo-Repo M: Inadequately low serum levels of steroid hormones in relation to interleukin- 6 and tumor necrosis factor in untreated patients with early rheumatoid arthritis and reactive arthritis. Arthritis Rheum 2002;46:654-662.

12 Perez AR, Silva-Barbosa SD, Berbert LR, Revelli S, Beloscar J, Savino W, et al: Immunoneuroendocrine alterations in patients with progressive forms of chronic Chagas disease. J Neuroimmunol 2011;235:84-90.

13 dos Santos VM, da Cunha SF, Teixeira V de P, Monteiro JP, dos Santos JA, dos Santos TA, et al: Frequency of diabetes mellitus and hyperglycemia in chagasic and non-chagasic women. Rev Soc Bras Med Trop 1999;32:489_ 496.

14 Cabalén ME, Cabral MF, Sanmarco LM, Andrada MC, Onofrio LI, Ponce NE, et al: Chronic Trypanosoma cruzi infection potentiates adipose tissue macrophage polarization toward an anti-inflammatory M2 phenotype and contributes to diabetes progression in a diet-induced obesity mode. Oncotarget 2016; 7:13400-13415

15 Nagajyothi F, Desruisseaux MS, MacHado FS, Upadhya R, Zhao D, Schwartz GJ, et al: Response of adipose tissue to early infection with trypanosoma cruzi (Brazil Strain). J Infect Dis 2012;205:830-840.

16 Nagajyothi F, Desruisseaux MS, Thiruvur N, Weiss LM, Braunstein VL, Albanese C, et al: Trypanosoma cruzi Infection of cultured adipocytes results in an inflammatory phenotype. Obesity 2008;16:1992-1997.

17 Cabalén ME, Cabral MF, Sanmarco LM, Andrada MC, Onofrio LI, Ponce NE, et al: Chronic Trypanosoma cruzi infection potentiates adipose tissue macrophage polarization toward an anti-inflammatory M2 phenotype and contributes to diabetes progression in a diet-induced obesity model. Oncotarget 2016; 7:13400-13415.

18 Combs TP, Nagajyothi, Mukherjee S, De Almeida CJ, Jelicks LA, Schubert W, et al: The adipocyte as an important target cell for Trypanosoma cruzi infection. J Biol Chem 2005; 280:24085-24094.

19 Tanowitz HB, Jelicks LA, Machado FS, Esper L, Qi X, Desruisseaux MS, et al: Adipose tissue, diabetes and chagas disease. Adv Parasitol 2011;76:235-250.

20 Ferreira AVM, Segatto M, Menezes Z, Macedo AM, Gelape C, de Oliveira Andrade L, et al: Evidence for Trypanosoma cruzi in adipose tissue in human chronic Chagas disease. Microbes Infect 2011;13:1002-1005.

21 Barbosa-Ferreira JM, Mady C, Ianni BM, Lopes HF, Ramires FJA, Salemi VMC, et al: Dysregulation of autonomic nervous system in chagas' heart disease is associated with altered adipocytokines levels. PLoS One 2015; 10:e0131447.

22 Garzon SA, Lorga AM, Nicolau JC: Electrocardiography in Chagas' heart disease. Sao Paulo Med J 1995;113:802-813.

23 Alberti KGMM, Zimmet P, Shaw J: Metabolic syndrome-a new world-wide definition. A
Consensus Statement from the International Diabetes Federation. Diabet Med 2006;23: 469-480.

24 Alberti KGMM, Zimmet P, Shaw J: The metabolic syndrome - a new worldwide definition. Lancet 2005;366:1059-1062.

25 Törüner F, Akbay E, Cakir N, Sancak B, Elbeg $S$, Taneri F, et al: Effects of PPARgamma and PPARalpha agonists on serum leptin levels in diet-induced obese rats. Horm Metab Res 2004;36:226-230.

26 Jiang C, Ting AT, Seed B: PPAR-gamma agonists inhibit production of monocyte inflammatory cytokines. Nature 1998;391:8286.

27 Yang XY, Wang LH, Chen T, Hodge DR, Resau JH, DaSilva L, et al: Activation of human $\mathrm{T}$ lymphocytes is inhibited by peroxisome proliferator-activated receptor gamma (PPARgamma) agonists. PPARgamma co-association with transcription factor NFAT. J Biol Chem 2000;275:4541-4544.

28 Geraix J, Ardisson LP, Marcondes-Machado J, Pereira PC: Clinical and nutritional profile of individuals with Chagas disease. Braz J Infect Dis 2007;11:411-414.

29 Compagnucci AB, Dávila A, Beloscar J, Pezzotto SM, Dávila H: Ingesta alimentaria y estado nutricional de pacientes con enfermedad de Chagas. Arch Latinoam Nutr 2016;66:185194.

30 Martí A, Marcos A, Martínez JA: Obesity and immune function relationships. Obes Rev 2001;2:131-140.

31 Milner JJ, Beck MA: The impact of obesity on the immune response to infection. Proc Nutr Soc 2012;71:298-306.

32 Feuerer M, Herrero L, Cipolletta D, Naaz A, Wong J, Nayer A, et al: Lean, but not obese, fat is enriched for a unique population of regulatory $\mathrm{T}$ cells that affect metabolic parameters. Nat Med 2009;15:930-939.

33 Lumeng CN, Delproposto JB, Westcott DJ, Saltiel AR: Phenotypic switching of adipose tissue macrophages with obesity is generated by spatiotemporal differences in macrophage subtypes. Diabetes 2008;57:32393246.

34 Strissel KJ, DeFuria J, Shaul ME, Bennett G, Greenberg AS, Obin MS: T-cell recruitment and Th1 polarization in adipose tissue during diet-induced obesity in C57BL/6 mice. Obesity (Silver Spring) 2010;18:1918-1925. 
35 Weisberg SP, McCann D, Desai M, Rosenbaum M, Leibel RL, Ferrante AW: Obesity is associated with macrophage accumulation in adipose tissue. J Clin Invest 2003;112:17961808.

36 Skurk T, Alberti-Huber C, Herder C, Hauner $\mathrm{H}$ : Relationship between adipocyte size and adipokine expression and secretion. J Clin Endocrinol Metab 2007;92:1023-1033.

37 Moriyama Y, Yasue H, Yoshimura M, Mizuno $Y$, Nishiyama K, Tsunoda R, et al: The plasma levels of dehydroepiandrosterone sulfate are decreased in patients with chronic heart failure in proportion to the severity. J Clin Endocrinol Metab 2000;85:18341840.

38 Santos CD, Toldo MP, Santello FH, Filipin Mdel V, Brazao V, do Prado Junior JC: Dehydroepiandrosterone increases resistance to experimental infection by Trypanosoma cruzi. Vet Parasitol 2008;153:238-243.

39 Roggero E, Pérez AR, Tamae-Kakazu M, Piazzon I, Nepomnaschy I, Basedovsky HO, et al: Edogenous glucocorticoids cause thymus atrophy but are protective during acute Trypanosoma cruzi infection. J Endocrinol 2006; 190:495-503.

40 Myers MG, Leibel RL, Seeley RJ, Schwartz MW: Obesity and leptin resistance: Distinguishing cause from effect. Trends Endocrinol Metab 2010;21:643-651.

41 Ouchi N, Shibata R, Walsh K: Cardioprotection by adiponectin. Trends Cardiovasc Med 2006; 16:141-146.

42 Mahajan R, Lau DH, Sanders P: Impact of obesity on cardiac metabolism, fibrosis, and function. Trends Cardiovasc Med 2015;25: 119-126.
43 Tanowitz HB, Amole B, Hewlett D, Wittner M: Trypanosoma cruzi infection in diabetic mice. Trans R Soc Trop Med Hyg 1988;82: 90-93.

44 Fernandes F, Dantas S, Ianni BM, Ramires FJ a, Buck P, Salemi VMC, et al: Leptin levels in different forms of Chagas' disease. Braz J Med Biol Res 2007;40:1631-1636.

45 Karmazyn M, Rajapurohitam V: Leptin as a cardiac pro-hypertrophic factor and its potential role in the development of heart failure. Curr Pharm Des 2014;20: 646-651.

46 Schulze PC, Kratzsch J, Linke A, Schoene N, Adams V, Gielen S, et al: Elevated serum levels of leptin and soluble leptin receptor in patients with advanced chronic heart failure. Eur J Heart Fail 2003;5:33-40.

47 Pereira Barretto AC, Mady C, Arteaga-Fernandez E, Stolf N, Lopes EA, Higuchi ML, et al: Right ventricular endomyocardial biopsy in chronic Chagas' disease. Am Hear J 1986; 111:307-312.

48 Bornstein SR, Uhlmann K, Haidan A, Ehrhart-Bornstein M, Scherbaum WA: Evidence for a novel peripheral action of leptin as a metabolic signal to the adrenal gland: leptin inhibits cortisol release directly. Diabetes 1997;46:1235-1238.

49 Shen Y, Charlesworth JA, Kelly JJ, Loi KW, Peake PW: Up-regulation of adiponectin, its isoforms and receptors in end-stage kidney disease. Nephrol Dial Transplant 2007;22: 171-178.

50 Tan W, Wang F, Zhang M, Guo D, Zhang Q, He S: High adiponectin and adiponectin receptor 1 expression in synovial fluids and synovial tissues of patients with rheumatoid ar- thritis. Semin Arthritis Rheum 2009;38:420427.

51 Peschon JJ, Torrance DS, Stocking KL, Glaccum MB, Otten C, Willis CR, et al: TNF receptor-deficient mice reveal divergent roles for p55 and p75 in several models of inflammation. J Immunol 1998;160:943-952.

52 Minigo G, Woodberry T, Piera KA, Salwati E, Tjitra E, Kenangalem E, et al: Parasite-dependent expansion of TNF receptor II-positive regulatory $\mathrm{T}$ cells with enhanced suppressive activity in adults with severe malaria. PLoS Pathog 2009;5:e1000402.

53 Leininger MT, Portocarrero CP, Houseknecht KL: Peroxisome proliferator-activated receptor gammal expression in porcine white blood cells: dynamic regulation with acute endotoxemia. Biochem Biophys Res Commun 1999;263:749-753.

54 Soller M, Tautenhahn A, Brüne B, Zacharowski $\mathrm{K}$, John S, Link $\mathrm{H}$, et al: Peroxisome proliferator-activated receptor gamma contributes to $\mathrm{T}$ lymphocyte apoptosis during sepsis. J Leukoc Biol 2006;79:235-243.

55 Penas FN, Carta D, Dmytrenko G, Mirkin GA, Modenutti CP, Cevey ÁC, et al: Treatment with a new peroxisome proliferator-activated receptor gamma agonist, pyridinecarboxylic acid derivative, increases angiogenesis and reduces inflammatory mediators in the heart of Trypanosoma cruzi-infected mice. Front Immunol 2017;8:1738.

56 Stefanou N, Satra M, Papanikolaou V, Kalala F, Gatselis N, Germenis A, et al: Leptin receptor isoforms mRNA expression in peripheral blood mononuclear cells from patients with chronic viral hepatitis. Exp Biol Med (Maywood) 2006;231:1653-1663. 